\title{
Supporting Implicit Human-to-Vehicle Interaction: Driver Identification from Sitting Postures
}

\author{
Andreas Riener and Alois Ferscha \\ Johannes Kepler University Linz, Institute for Pervasive Computing \\ Altenberger Str. 69, A-4040 Linz, Austria \\ Tel. +43/732/2468-8555, Fax. +43/732/2468-8524
}

E-mail: \{riener,ferscha\}@pervasive.jku.at

\begin{abstract}
Mobile internet services have started to pervade into vehicles, approaching a new generation of networked, "smart" cars. With the evolution of in-car services, particularly with the emergence of services that are personalized to an individual driver (like road pricing, maintenance, insurance and entertainment services) the need for reliable, yet easy to handle identification and authentication has arisen. Services that demand unambiguous and unmistakable continuous identification of the driver have recently attracted many research efforts, mostly proposing video-based face/pose recognition, or acoustic analysis.

A driver identification system for vehicular services is proposed, that, as opposed to video or audio based techniques, does not suffer from the continuously changing environment while driving, like lighting or noise conditions. A posture recognition technique based on a high resolution pressure sensor integrated invisibly and unobtrusively into the fabric of the driver seat has been developed, taking the pelvic bone distance as a biometric trait. Data coming from two $32 x 32$ pressure sensor arrays (seat- and backrest) is classified according to features defined based on the pelvic bone signature, mid and high pressure distribution and body weight. Empirical studies, besides analyzing (quantitative) driver recognition performance, assess the identification technique according to the qualitative attributes universality, collectability, uniqueness, and permanency.

The proposed driver identification technique is implicit and thus not reliant to attention, it is continuously in operation while seated, and requires no active person cooperation.
\end{abstract}

Permission to make digital or hard copies of all or part of this work for personal or classroom use is granted without fee provided that copies are not made or distributed for profit or commercial advantage and that copies bear this notice and the full citation on the first page. To copy otherwise, to republish, to post on servers or to redistribute to lists, requires prior specific permission and/or a fee.

ISVCS 2008, July 22 - 24, 2008, Dublin, Ireland.

Copyright 2008 ACM ISBN 978-963-9799-27-1 ...\$5.00.
These characteristics encourage the universal use of the approach - a whole new modality for person-to-environment interaction seems possible.

Keywords. Continuous Person Identification, Sitting Postures, Implicit Interaction, Interaction modalities.

\section{Motivation}

Due to the evolution of mobile wireless communication technologies, and the explosive growth of the internet itself, the vision of "internet enabled cars" has become a reality. An ample effort in research and development has been undertaken by stakeholders in the automotive industry, and even political authorities. Among the most prominent recent research challenges are "intelligent networked car solutions" ("Intelligent Car Initiative"1), identifying research areas like "next generation driver assistance systems", "cooperative systems based on C2C (Car-to-Car) and C2I (Carto-Infrastructure) communication" and "real-time traveller and traffic information"[5]. Numerous scenarios motivate the improvements and advantages that could be attained regarding to safety, security, comfort and even ejoyability, if services delivered to cars would be based on notions of context, knowledge and "intelligence", while the underpinning technology of "Intelligent Cars" is identified as being communications: "[..]interaction between the driver, the vehicle and the road environment in an integrated approach[..]"[5].

In order to implement personalization at the services and applications level, reliably, trustworthy, unambiguous and continuous identification mechanisms have been identified as a key enabling technology [9] for services in networked cars. Reliably identifying the driver of a vehicle with technologies not prone to misuse (like sharing ignition keys, swapping the person who drives, etc.) is an indispen-

\footnotetext{
${ }^{1}$ European Commission i2010 Intelligent Car Initative, URL: http://ec.europa.eu/information_society/ activities/intelligentcar, last visited: May 18, 2008
} 


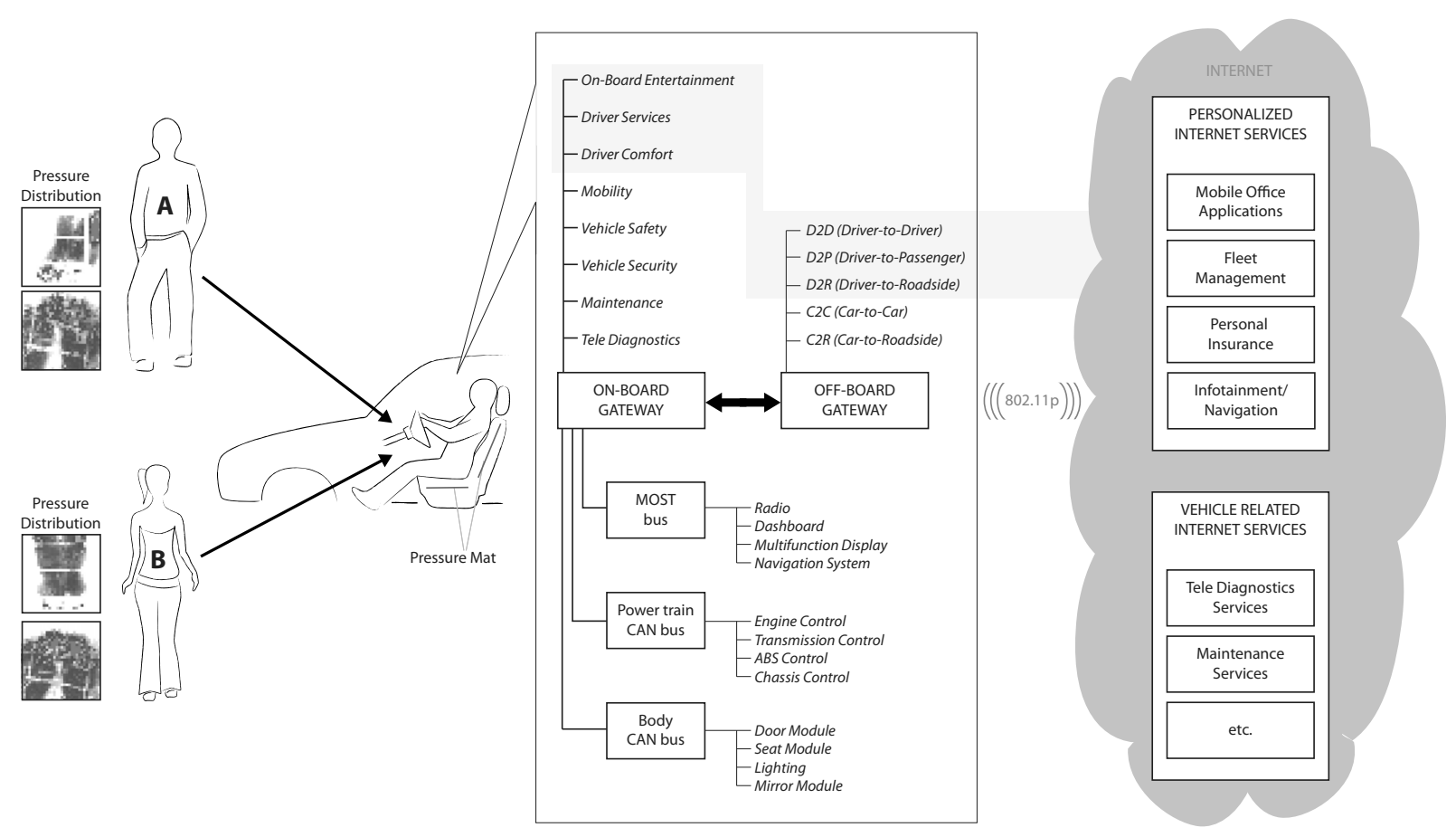

Figure 1. Driver identification from sitting postures and examples of personalized vehicular services

sible prerequisite for services involving accounting or billing like dynamic road pricing, dynamic insurance pricing, cause based taxation or consumer controlled entertainment.

As for example, a system for calculating a personalized car insurance rate based on the driving-behavior of a person has been proposed in [3] and [4]. Several options for drivers to attack the insurance system and modifying insurance rates, e.g. by software adaptation, replacing of communication messages, modify in-car sensor values, etc. have been discussed. Although insurance rate calculation is proposed to be personalized, the driver itself is not inspected - he is simply identified as being the owner of the car; but usually a couple of persons (e.g. family members) share one car. If the insurance contract is person-bound, and person identification is done e.g. by personal ignition or pincode, this identification could be outwitted. An example scenario of abuse could be the following: A person "A" which is in an aggressive driving state could identify itself as person "B", drives then with high risk or even drunk, violates speed limits or ignores red lights, etc. and thereby automatically increases the insurance rate of "B", while "A"s own insurance contract will not be affected.

To solve such problems, personalized, networked car applications require a solution for safe person identification to guarantee that person-bound services in cars are effectivly linked to the subject concerned. A number of biometric identification methods applicable in the automotivefield have been introduced. Most of them require active participation of the involved user, e.g. sensors located in the car door handles, fingerprint readers inside the car $[15$, p.60], retina or iris scan systems. Other systems identify the driver by a unique key, e.g. by pressing a "personal" button beside the seat or using a RFID-tag embedded in a personalized ignition key.

Traditional approaches of identification are not based on any inherent attribute of an individual; they suffer from a number of obvious disadvantages: A key can be lost or stolen, a pincode may be forgotten or guessed by an impostor [11, p.91] and then permits unallowed persons to control the vehicle (unauthorized handover). On the other hand, RFID-technology for identification is antiquated and insufficient for offering stable, reliable, authentic and trustworthy individual-related services ${ }^{2}$.

In this paper we propose a implicit "sitting posture recognition system", implemented as a passive method that inspects the pressure patterns on driver's seat and backrest.

A few more ideas of applications in networked cars which would gain from our approach of driver identification are the following:

(i) Cause-based taxation: Climate change and pollution has raised consideration of political authorities to cause-based taxation models for drivers. Whether the car nor its owner should be the subject of emission regulations (like exhaust, green house, $\mathrm{CO}_{2}$ ), but the individual driver. Legislation for cause-based taxation would be ultimately reliant to reliable driver identification.

\footnotetext{
'2Analyzing RFID's Reliability And Stability Limitations, URL: http://www.informationweek.com/industries/ showArticle.jhtml?articleID=53200075 (releasing date: November 16, 2004)
} 
(ii) Personalized insurance policies: In the last 50 years a steadily increasing volume of traffic (caused by flexible work schedules, longer journeys to the place of work, and the demand of people for a more mobile lifestyle) has been discovered [1]. This trend is set to continue [18], causing more traffic jams and a higher risk of travelling on roads [9].

The future of mobility will be different compared to todays individual traffic: Public transportation will gain importance in the near future by providing fast point to point connections between urban centers with airplanes, trains or busses driving on individual lanes. Only the last few kilometers from airports or railway stations to the destination outside cities will be driven with rental or shared cars. Highly flexibly models for covering this picture are offered already today, e.g. by Deutsche Bahn AG or ÖBB in Austria. Different types of cars can be rented by the hour, day or week without paying basic charges. Pick-up and return is possible in a lot of cities all around the country (200 locations in Austria $^{3}$ ), at any time of day or night.

Car rental fees, particulary dominated by insurance rates, are quite high today. A careful and accidentfree driver which frequently uses car-sharing would suppose a personal insurance rate (which of course is cheaper than the actual one from the rental-car provider). The contract would be bound to the individual and no longer to the car or the car owner and would automatically carried with the driver and applied to any driven car ("cross-car" insurance).

(iii) Logistics and fleet management: (i) Delivery vans of small transport companies (pharmaceutical product transports, home lunch services, etc.) are steered by multiple persons. (ii) In heavy load transportations a few lorry drivers share one and the same truck. (iii) Most taxi companies operate a vehicle fleet. Cabs are operated 24 hours a day by different people.

In all these cases administrative costs could be minimized and misusage could be avoided if the operator is aware of the actual driver of any vehicle. Secondary, the use of personal insurance contracts from each driver (as described above) could minimize running costs of each car or truck (but on the other hand, for persons with high insurance rates, e.g. poor drivers, it would be hard to get a driving job).

(iv) Car comfort: Networked driver identification leads to auto-configuration of any vehicle at boarding-time. This is particulary comfortable if the car is shared or interchanged by a number of persons (e.g. family members), or for any car-rental model.

Beside personalized services in networked car environments, person identification from sitting postures also generate surplus value in single-car applications:

(i) Safety: Authorization by using sitting postures as alternative or add-on to common ignition keys. Only

\footnotetext{
${ }^{3}$ http://www.denzeldrive.at/carsharing/142_293.php,
} (last visited: May 18, 2008) permitted persons are allowed to drive the car. Beside this, the feature directs to respectable limitations. What is, for instance, when the owner of the car is injured or incapacitated, and someone else must drive him to a hospital - has the authorization process be completed before the helping person could use the vehicle?

(ii) Intelligent driver assistance: Select voice type of the navigation system, manage the air-conditioning system, choose the designated radio station, control background-lightning of dashboard, etc. according to users bias, and maybe enhanced by additional sensors (such as ECG-device, respiration sensor, GPS unit).

(iii) Vehicle dynamics control: Automatically adapt the cars running gear corresponding to users preferred driving behaviour or evidenced assumptions (soft running gear for family trips, tough sports suspension in a race, etc.).

(iv) Implicit interaction: Handicapped persons would benefit from alternatives to common input devices, such as switch or leverage, if using specific pressure patterns, not appearing in common driving situations, and mapped to pre-defined actions/activities. For example, a turn is forthwith not done by operating the direction indicator/leverage, but by pressing the left shoulder twice into the back of the seat.

(v) Driving comfort: Automatically adapt parameters of the car (height and offset of the seat, adjust mirrors, etc.) if a person is identified.

The performed studies and enforcement of experiments followed the suggestions of Jain et. al. [11, p. 92], which recommended the four characteristics universality, collectability, uniqueness and permanency as beeing essential for good and accurate biometric systems. In our tests, these attributes are analyzed isolated to get more meaningful results whether they are suitable for unique distinction of any person (and therefore allows in-car person identification) or not.

Outline: The rest of the paper is structured as follows: Section 2 motivates experimental system design, section 3 gives an introduction and explanation to the referred biometric characteristics. Section 4 describes the setup of our testbed for body-posture sensing. Section 5 mentions the features, extracted from the pressure mats and utilized for data evaluation and processing, section 6 gives a presentation of the achieved results for driver identification. The last section addresses our findings and encountered problems, and suggests future work.

\section{Driver Identification Systems}

As already mentioned above, a lot of research has been conducted in the area of $\mathrm{C} 2 \mathrm{C}$ and $\mathrm{C} 2 \mathrm{I}$. Various specialized communication (e.g. 802.11p) and sensor technologies as well as application scenarios have been developed - to 
give a summary over the wide range of topics and technologies is nearly impossible (the main subjects are: wireless communication, security, reliability, driver assistance systems). Initatives are partly funded by research ministries all around europe as well as european commission.

Further interestingness and actuality of this topic can be indicated by large industry consortiums like Germany's BMW AG and Volkswagen AG, France's Renault, Italy's Fiat SpA, etc. and a number of research institutes like Fraunhofer, NEC Europe Ltd., Siemens AG or Bosch GmbH doing research in this domain (project pages available on the internet presents foci and results of the individual cooperations).

Andreoni et. al. [2] had already presented a system for analyzing postures of car drivers in 2002. They noticed, that "[..]it is evident that an integrated analysis of all the aspects involved in the car driver posture is very complex and practically impossible to propose[..]" [2, p.512]. In [6], an overview about pressure mapping systems and their usage is given. [10] introduces a model for extracting postures from humans in motion, [12] discusses the usage of sitting postures and measurements with FSA-mats, [19] concentrates on the identification of static sitting postures. Overbeeke et.al. [13] and R. Picard together with her research group presented several approaches of using FSA-mats in traditional office-chairs to identify distinct user-states, e.g. in [16] and [8].

None of the discussed approaches had been evaluated in the car-domain. Furthermore, they had not raised the claim of presenting a system for identifying persons by their sitting postures, and thus maybe brings our proposed system into the position of beeing the first one for unique distinguishing between any number of persons sitting on a car seat.

\section{Biometric Identification}

Biometric identification refers to identifying a person on the base of his or her physiological characteristics and biometric identifiers. Our experiments for driver identification from sitting postures are aligned along the recommendations for ideal biometric systems from Jain et. al. [11, p.92]. They also accounted that these requirements are not always feasible for useful biometric systems and that other issues had to be considered in the realization of an authentic system:

(i) Performance is, beside factors like wastage of resource, characterized by the attributes false reject-rate and false-alarm rate [15, p.57]. In a optimal (biometric) system both of these error rates tend to zero.

(ii) Acceptability denounces the willingness of people to accept the usage of a particular biometric identifier in everyday life.

(iii) Circumvention means, how easy it is to fool the system through fraudulent methods.

The significance of any biometric identification system is determined by the assortment of appropriate features. According to our considerations above, following characteristics have to be validated in our experimental setting: (i) Universality - "Every person should have this feature": In the current experiment we only use pressure disseminations from seat- and backrest-mat. Each driving person has a bottom and a back, therefore all selected features (specification follows later in section 5) are collectable.

(ii) Collectability - "It should be possible to extract the interested attributes": Any car driver sit's on the seat, and consequently produces pressure patterns on seat- and backrest-mat. (At least, if the person sit's on the leading area of the seat or is leaning forward, pressure is indicated on the seat mat, but not on the backrest mat).

(iii) Uniqueness - "There should be only one person with this characteristics": For the indication of uniqueness it is necessary to train a test database with a large number of subjects. We endorse this suggestion with additional restraints of measured people: They should be in a tight belt of age (20 to 30 years old) and should have the same stature (height from 175 to $185 \mathrm{~cm}$, weight from 70 to $80 \mathrm{~kg}$ ) to avoid dependencies and divergence.

(iv) Permanency - "The features should always stay the same": Different clothes (beach wear, jeans, skioverall), trouser buttons pressing on the mat, or objects in the back-pocket (wallet, cell phone, bunch of keys) affect the results ("white noise"). For considering problems regarding the feature permanency, a special experiment with different artefacts in the pockets has been done.

\section{Experimental Setup for Body Pos- ture Sensing}

For data acquisition in our prototype (see Figure 2) we used force sensor arrays (FSA), interconnected to a common notebook computer with USB interfaces. The proposed system is universal and could be used in any type of car (utility-driven car, sports car with body-contoured seats, comfort station wagon, etc.) for arbitrary style of sitting or driving (rush-hour traffic, family trip, etc.).

\section{Research Hypothesis}

We suppose that the 4 biometric characteristics, mentioned just above, are proper to discriminate sitting postures of any person inside a car. Experimental settings in different types of cars (sports car, family van, etc.) and with a large number of subjects has to be processed to approve or reject this hypothesis.

\section{Pressure Mapping: Requirements}

A pressure mat system has to fulfill the following needs to be appropriate for us: (i) The area of common seats in a car is about $50 * 50 \mathrm{~cm}^{2}$, thus the size of utilized force sensor arrays should be similar. The mat itself should be flexible and thin so that it's easy deployable in a car; (ii) The system should be able to be applied to any person with possibility of driving a car, e.g. persons from 18 up to 75 years 

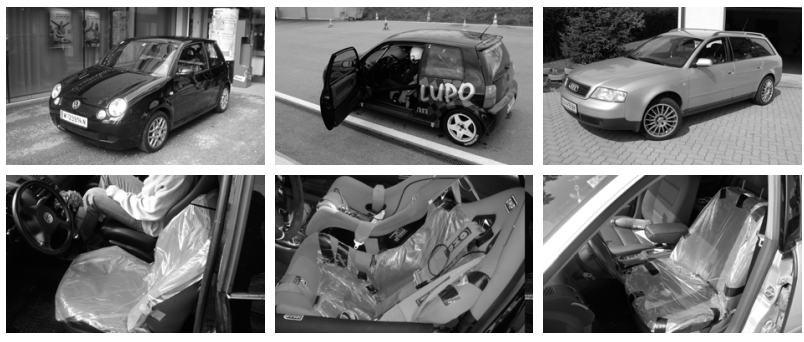

Figure 2. Driver identification prototype, installed in utility car, sports car and comfort station wagon (from left to right)

and a weight of 40 to about $125 \mathrm{~kg}$; (iii) Precise measurements with a large number of sensors should be possible with a high update rate of less than one second. Three systems matching these requirements had been identified; we have decided to use the "FSA Dual High Resolution System" from Vista Medical against the other two systems (Tekscan, XSENSOR) (because of a local technical consultant of the first one).

The selected system allows recording of the loads on a thin, flexible sensor-mat, consisting of a matrix of 32 by 32 piezoresistive sensors. Each of them covers a range of 0 to $26,67 \mathrm{kPa}$ (data specification of the mat system indicates a pressure range from 0 to $200 \mathrm{~mm}_{H g}$, but as $k P a$ is a SI unit, all Torr or $m m_{H g}$ values are conversed). The sampling rate of a mat is normally in the range 1 to $10 \mathrm{~Hz}$, but sometimes connection errors lead to a slight delay.

\section{Sensing Software}

The original pressure sensing system as shipped with had to be ported to better integrate into the evaluation environment. Particularly, a data acquisition and evaluation application had to be implemented. Communication with the data collection system was then implemented based on the OLE components included in the FSA software package.

Improve Sensor Readings. Because of sudden movements and other artefacts, it is necessary to generate a more sophisticated matrix of sensor readings. This has been done by calculating the median of each of the 1.024 sensor values over a series of measurements (we preferred the "median" against the "mean" because of it's better stability concerning to outliers). Tests showed us that 5 measurements are enough to create a sufficiently stable matrix. To guarantee data integrity and avoid errors in measurement, each experiment has been repeated and stored at least 4 times (in consideration of the hamming distance). Pelvic bones of male and female are different and allows gender discrimination (e.g. as showed in [14]), hence we concentrated our actual experiments primarily on male subjects ( 27 persons out of the whole of 34 subjects), the class of female drivers should be reliable distinguished from the male ones by evaluation of pelvic bones.

Reference Weight. After calibration, a reference weight has been applied to the seat mat. This had been done by placing a specific person (with given weight) onto the seat mat and performing 100 consecutive readings from it. The mean value of the accumulated pressures is used as reference factor for later weight estimations.

Subjects. For a validation of our hypothesis (see section 4 above), the experiment was applied to a total of 34 persons as shown in Table 1, most of them were male and $80 \%$ $\left(P_{90}-P_{10}\right)$ were in the age from 20 to 31 years, acquired on the university campus and around. Male subjects vary in weight from $58 \mathrm{~kg}$ to $104 \mathrm{~kg}$, and in size from $160 \mathrm{~cm}$ to $194 \mathrm{~cm}$. The 7 female test persons vary from $46 \mathrm{~kg}$ to $70 \mathrm{~kg}$ in weight, and from $160 \mathrm{~cm}$ to $173 \mathrm{~cm}$ in size. For easier evaluations, all of them were wearing long trousers with no artefacts (wallet, cell phone, etc.) in the back-pockets. Each subject had been briefed to adjust the seat to sit comfortable in the car, reaching clutch and throttle pedal and put both hands onto the steering-wheel (driving-position). Changing the pre-defined position of the backrest had not been allowed, hence the angle between backrest and seat was equal for any reading.

\section{Test Execution}

To assess the quality and accuracy of the current prototype, a database with pressure patterns from 34 subjects had been created. All tests had been processed inside a comfort station wagon (see right picture in Figure 2) with pressure mats attached to both, seat and back. We used euclidean distance metrics, a well-researched approach in the field of pattern recognition, to match a persons current pressure dissemination against earlier stored patterns. Execution of the experiment had been done in two stages:

Training set. In the first phase, a large number of data samples had been captured, features of the test persons had been extracted and stored into a database: Some personal data had been collected from each test person (Nickname, Age, Gender, Weight and Size) and stored additionally. After that, consecutive reading of sensor data is started, alternating between seat and backrest mat; raw-data is written into the database, completed by a timestamp and personal data. The estimated duration for recording of one person is less than 5 minutes, including introduction and briefing.

The prototype has been used in different types of cars (respectively on different types of seats), for meaningful interpretations only the data sets from one type (Audi A6, rightmost-images in Figure 2) had been used. By evaluating the pressure distribution on the seat mat, weight of subjects could be estimated.

Testing set. Afterwards (in the "live system") the sample of any acquired person has been compared to all stored data sets to locate the best matching dataset (they are represented by most similar pressure disseminations).

\section{Feature Determination, Classification, and Mapping}

Subsequent listed features have been selected and used 


\begin{tabular}{||l|r|r|r|r|r|r|r|r|r||}
\hline Sex & $\begin{array}{r}\text { Number } \\
\text { Subjects }\end{array}$ & $\begin{array}{r}\text { Percentage } \\
\%\end{array}$ & $\begin{array}{r}\text { Min } \\
x_{\min }\end{array}$ & $\begin{array}{r}\text { Max } \\
x_{\max }\end{array}$ & $\begin{array}{r}\text { Mean } \\
\bar{x}\end{array}$ & $\begin{array}{r}\text { Median } \\
\widetilde{x}\end{array}$ & $\begin{array}{r}\text { Std.Dev. } \\
\sigma\end{array}$ & $\left(P_{10}\right)$ & $\left(P_{90}\right)$ \\
\hline \multicolumn{10}{|c||}{ Age } \\
\hline All & 34 & 100.00 & 19.00 & 53.00 & 25.35 & 24.50 & 6.17 & 20.00 & 30.70 \\
Female & 7 & 20.59 & 19.00 & 53.00 & 30.57 & 28.00 & 7.90 & 22.60 & 40.40 \\
Male & 27 & 79.41 & 19.00 & 31.00 & 24.00 & 23.00 & 12.45 & 20.00 & 28.80 \\
\hline \multicolumn{10}{|c||}{ Size } \\
All & 34 & 100.00 & 160.00 & 194.00 & 165.65 & 178.00 & 10.78 & 168.00 & 185.70 \\
Female & 7 & 20.59 & 160.00 & 173.00 & 165.86 & 168.00 & 5.67 & 160.00 & 171.20 \\
Male & 27 & 79.41 & 168.00 & 194.00 & 179.44 & 180.00 & 8.74 & 171.80 & 186.00 \\
\hline \multicolumn{10}{|c||}{ Weight } \\
\hline All & 34 & 100.00 & 46.00 & 104.00 & 72.00 & 73.00 & 3.50 & 56.60 & 85.00 \\
Female & 7 & 20.59 & 46.00 & 70.00 & 58.00 & 56.00 & 5.69 & 49.60 & 69.40 \\
Male & 27 & 79.41 & 58.00 & 104.00 & 75.63 & 75.00 & 10.61 & 63.00 & 85.00 \\
\hline
\end{tabular}

Table 1. Sex-related statistics of subjects used in the experiment

for our evaluations. Futhermore, suggestions for choosing this features are accounted.

(i) Pelvic Bones: This complex feature includes the location of the pelvic bones on the seat mat. They produce the highest amount of pressure on the seat, which could be detected by the position of the two regions on the mat with maximum pressure value. An example of how this could be found is shown in Figure 3. In our current implementation, euclidian distance between the two points with the highest pressure is calculated and used for further evaluations.

A. Durability: The distance of pelvic bones does not change if we gain weight or wear different clothes. Thus, this feature is permanent.

B. Sex-dependency: Females have a differently shaped pelvic than males. The possibility of discrimination has been identified and published early, e.g. in [7] and [17].

(ii) Mid to High Pressure Area: The value for this feature is calculated from the number of sensors where it's load is higher than $10 \%$ of maximum pressure force indicated on the mat. In Figure 3, amount of this feature is equivalent to the number of non-white pixels.

(iii) High Pressure Area: This feature is the area of pressure cells with values exceeding $90 \%$ of the maximum pressure measured on the mat. It can be seen as the dark-grey surface in Figure 3.

(iv) Weight: Is calculated as accumulation of pressure values, measured on any of the 1.024 sensors. By the reason that every sensor covers the same area we can simply add up the individual amounts. The result is a fairly constant indicator for the weight of the current person. As explained later, we do not get an exact value for person's weight, it is a rather vague estimation; larger sensor mats and higher resolution could probably solve this problem.

The combination of the distinct features (i) to (iv) from above directs to the feature vector, which is stored into the database.

\section{Sitting Postures}

Due to the fact that drivers are sitting in many different postures, there were several enrollments for each person necessary (the pressure maps of different persons sitting in similar postures are on the contrary very much alike).

If a person "A" sits in a specific posture during the enrollment process, e.g. leaning forward, then everyone that sits later in a similar posture and has not been enrolled for that posture will also be identified as person "A". To avoid this, we specified specialized enrollment-rules as a fixed set of sitting postures. Each person has to be enrolled for all of this postures, in the current experiment only the posture "sit upright" had been used for evaluations.

\section{Permanent and Unique Features}

Although the bottom is a rather permanent part of the body, the pressure map we get is it not: It changes with every movement. This makes it hard to find features that stay stable. One feature that is independent of movement is the total pressure we exert on the ground. It changes in a linear fashion with the person's weight. Tests had shown, that with the currently hardware we get only a very vague estimation of the total pressure:

(i) Mat size: For a couple of test persons the seat mat is a little bit too small (in that case, part of the legs are lying outside the sensing surface).

(ii) Pressure range: At some points, especially at regions of pelvic bones, the pressure exceeds the calibration range maximum of the mat. Sensor values are cut at $26,67 \mathrm{kPa}$ and therefore a correct estimation of the total pressure on this area is not possible anymore.

(iii) Weight estimations: By reason of unbalanced load sharing and dead space between the sensors, the total weight could not be exactly estimated from the sum of all charged sensors.

\section{Training Datasets}

Basis for the identification process is a database with as many data sets as possible. The implemented enrollment 


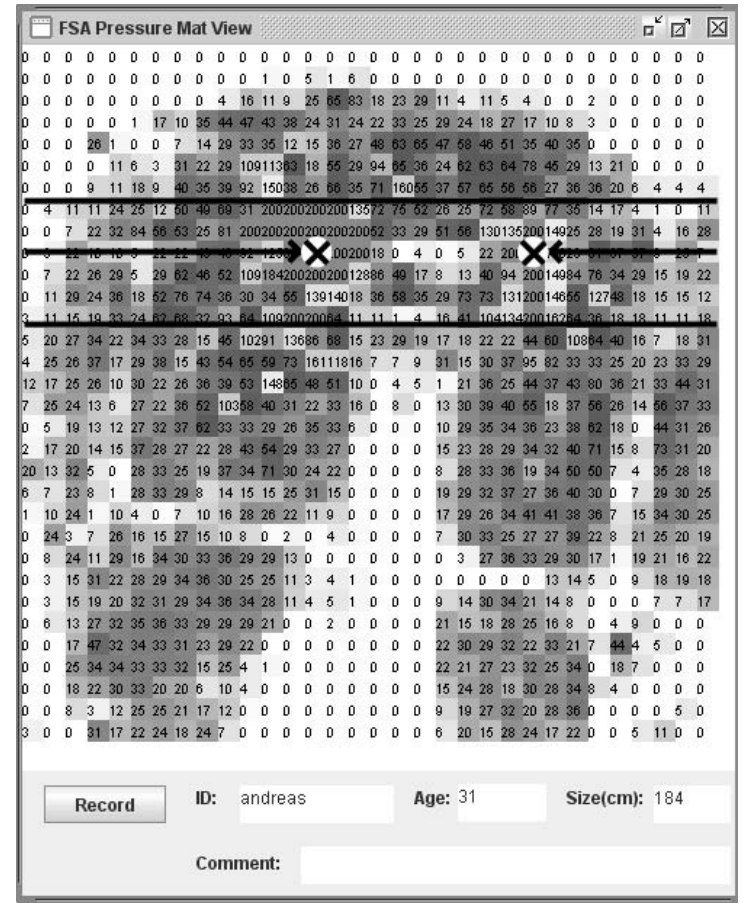

Figure 3. Pressure on the car seat with overlayed pelvic-bone identification

process determines the features of 5 different pressure maps, takes the median value of each and stores the resulting feature vector along with a timestamp and some personal data; statistical characteristics are calculated later. Although the driver of a car is severely limited in his movements (e.g. leaning to the side) there is still enough diversity that a single enrollment is unable to cover all aspects. The pressure distribution on the seat changes dramatically even with small movements - to encounter this, we enrolled every person at least 4 times.

\section{Classification and Mapping}

The identification process determines the feature vector of a new pressure mat reading and tries to find the closest vector in the database. Pairwise distances between all vector elements are calculated, multiplied with a weight factor and accumulated to the final indicator. The weight factors were obtained from experimental test series, with all features transformed in a "normalized space" to allow a meaningful comparison. Test series showed that most features perform almost identical, only the "High Pressure Area" turned out to be a rather poor indicator.

\section{Results}

The biometric identification features universality and collectability are trivially supported by our system. Consequently, we discuss the remaining characteristics permanency and uniqueness in more depth.

\section{Permanency}

Table 2 shows a confusion matrix of the seat mat for the experiment described in section 4 above. All sitting posture variantes ${ }^{4}$ of a subject with objects in the back-pocket are compared to each other. The upper triangular part of the confusion matrix shows the distinctions between postures with different artefacts. The scale factor was determined from the maximum difference of any two "normal" sitting postures, out of the 34 subjects in the database. The factor has been, independently for seat and back, set to $100 \%$. No artefacts have been directly placed on person's back, therefore variance on the backrest-mat is only little (maximum of $28.27 \%$, corresponding table is not shown) compared to variances on the seat mat. On the seat, the maximum difference for the pressure pattern between one subject in normal posture and the same person with applied artefacts in the back-pockets is $58.88 \%$. For reliable person identification this value is quite high, especially when compared to the maximum difference of any persons which is $100.00 \%$.

\begin{tabular}{||l|l|l|l|l|l|l|l|l||}
\hline & $\mathrm{N}$ & KL & KR & CL & CR & DL & DR & DRKL \\
\hline N & - & 29.4 & 39.8 & 50.0 & 46.5 & 55.6 & 52.7 & 58.9 \\
KL & & - & 38.3 & 42.6 & 43.0 & 48.4 & 49.9 & 51.0 \\
KR & & & - & 33.8 & 33.5 & 49.0 & 36.0 & 47.7 \\
CL & & & & - & 34.0 & 37.0 & 37.6 & 45.4 \\
CR & & & & & - & 36.9 & 28.8 & 39.5 \\
DL & & & & & & - & 41.8 & 34.3 \\
DR & & & & & & & - & 31.5 \\
DRKL & & & & & & & & - \\
\hline
\end{tabular}

Table 2. "Permanency-Test": Confusion matrix of postures with artefacts for the seat

Experimental Results. In the current prototype the feature permanency cannot be guaranteed if allowing any kind of artefacts in the pockets or even different clothes. Statistical characteristics of the artefact-afflicted datasets $\left(\min _{x}=\right.$ $\left.28.82 \%, \bar{x}=41.89 \%, \max _{x}=58.88 \%\right)$ are very similar to that of the normal datasets $\left(\min _{x}=24.78 \%, \bar{x}=54.79 \%\right.$, $\max _{x}=100.00 \%$ ). A more detailed comparison is given in Figure 4 as well as in Table 3 .

\section{Uniqueness}

For determining the accuracy and uniqueness of pressure patterns, the feature vector as described in section 5 has been evaluated for two subjects with a large number of recordings (31, 105 samples). Table 4 shows the results for the feature weight (raw data in the upper rows, normalized weights in the lower - factorized with the reference weight, as indicated in paragraph "Reference Weight" above). The mean-value gives a relative precise estimation of actual person's weight, but deviation is rather large. For evaluating

\footnotetext{
${ }^{4}$ Abbreviations for artefacts: N ... Normal posture, KL . Bunch of keys, left back-pocket, KR ... Bunch of keys, right back-pocket, CL ... Cell phone, left back-pocket, CR ... Cell phone, right back-pocket, DL ... Digital camera, left back-pocket, DR ... Digital camera, right back-pocket, DRKL ... Digital camera, right back-pocket and bunch of keys, left back-pocket
} 


\begin{tabular}{||l|l|l|l|l|l|l|l|l||}
\hline $\begin{array}{l}\text { Data } \\
\text { Sets }\end{array}$ & Database & $\begin{array}{l}\text { Min } \\
x_{\min }\end{array}$ & $\begin{array}{l}\text { Max } \\
x_{\max }\end{array}$ & $\begin{array}{l}\text { Mean } \\
\bar{x}\end{array}$ & $\begin{array}{l}\text { Median } \\
\widetilde{x}\end{array}$ & $\begin{array}{l}\text { Std.Dev. } \\
\sigma\end{array}$ & $\left(P_{25}\right)$ & $\left(P_{75}\right)$ \\
\hline \multicolumn{8}{|c||}{ Seat mat (normalized) } \\
\hline 34 & Normal & 24.78 & $100.00^{*}$ & 54.79 & 52.93 & 12.39 & 46.45 & 62.08 \\
8 & Artefacts & 28.82 & 58.55 & 41.89 & 40.79 & 8.13 & 35.59 & 48.75 \\
\hline \multicolumn{8}{||c||}{ Backrest mat (normalized) } \\
\hline 34 & Normal & 15.16 & $100.00^{*}$ & 41.10 & 38.43 & 12.00 & 32.21 & 45.56 \\
8 & Artefacts & 11.61 & 28.27 & 20.02 & 19.47 & 4.16 & 17.37 & 2.45 \\
\hline
\end{tabular}

*Maximum difference of any two normal datasets has been set to $100 \%$

Table 3. Data characteristics for boxplots of seat- and backrest-mats

\begin{tabular}{||l|l|l|l|l|l|l|l|l||}
\hline $\begin{array}{l}\text { Data } \\
\text { Sets }\end{array}$ & $\begin{array}{l}\text { Actual } \\
\text { Weight }\end{array}$ & $\begin{array}{l}\text { Min } \\
x_{\min }\end{array}$ & $\begin{array}{l}\text { Max } \\
x_{\max }\end{array}$ & $\begin{array}{l}\text { Mean } \\
\bar{x}\end{array}$ & $\begin{array}{l}\text { Median } \\
\tilde{x}\end{array}$ & $\begin{array}{l}\text { Std.Dev. } \\
\sigma\end{array}$ & $\left(P_{10}\right)$ & $\left(P_{90}\right)$ \\
\hline \multicolumn{8}{|c||}{ Weight } \\
\hline 105 & 75.5 & 137.976 & 196.339 & 176.589 & 177.599 & 14.530 & 157.749 & 193.004 \\
31 & 80.0 & 162.937 & 212.093 & 192.251 & 196.602 & 13.933 & 165.606 & 207.200 \\
\hline \multicolumn{8}{|c||}{ Weight (Normalized) } \\
\hline 105 & 75.5 & 58.797 & 83.667 & 75.251 & 75.682 & 6.192 & 67.223 & 82.246 \\
31 & 80.0 & 69.433 & 90.381 & 81.925 & 83.779 & 5.937 & 70.571 & 88.295 \\
\hline
\end{tabular}

Table 4. Accuracy of consecutive measurements of 2 subjects

the feature uniqueness, test candidates have been sat into the car again and a larger series of consecutive readings have been processed. For each of the tests measured postures have been compared to all patterns in the database and a list of deviations was stored in a separate table. The rank was assigned in the order of minimal differences - rank 1 means least deviation between current feature vector and the database-value (=best match), rank 34 means largest difference (=worst match, because database has exactly 34 datasets).

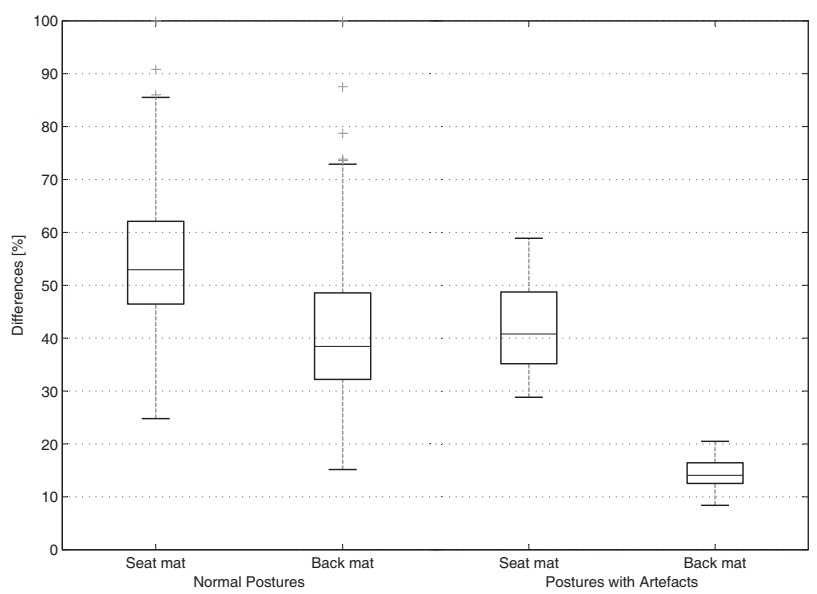

Figure 4. Boxplot comparing differences between the 34 "normal" datasets and that with "artefacts", each for seat and back

Experimental Results. A graphical representation of results can be found in Figure 5 (disjoined for the two experiments). The dark grey bars in the diagram shows that the matching algorithm for the 31 readings of experiment 1 directs to rank 1 to 6 . This means at worst case, which occurs in only $3.33 \%$, matching between a measurement against all 34 postures in the database results in place 6 . This stands for rather high stability for the sitting posture of that person. But on the other hand it can be seen, that exact match (=rank 1) is obtained in less than $25 \%$, which of course is poor. Light grey bars shows results for the $2^{\text {nd }}$ experiment, which is divergent to the first one: Exact match is here given in $48,08 \%$, but the remaining $51.92 \%$ are nearly even distributed to all ranks. $86,54 \%$ are assigned to ranks 1 to 10 , the remaining $13,46 \%$ are splitted into places 11 to 30 . Absolut worst case (which occurs in $0.96 \%$ ) is the classification into rank 30.

\section{Conclusions}

Motivated by an ample emergence of in-car services going way beyond the usual automatic customization of the vehicle's comfort configuration, to networked car services like mobile internet, dynamic road or dynamic insurance pricing, networked maintenance and entertainment services, etc., which all are reliant to unambiguous and unmistakable, continuous driver authentication, we have developed a driver identification method based on the biometrics of sitting.

Our approach is based on implicit interaction, inspecting sitting postures, acquired from pressure mats on driver's seat- and backrest. Posture recognition, opposed to vision detection techniques, does not suffer from environment conditions like brightness or weather. The measuring system itself is invisibly and unobtrusively integrable into the vehicle seat and avoids attentive participation of the user. In an experimental setup we have evaluated our system against the features of biometrical identification systems like universality, collectability, permanency, and uniqueness. 


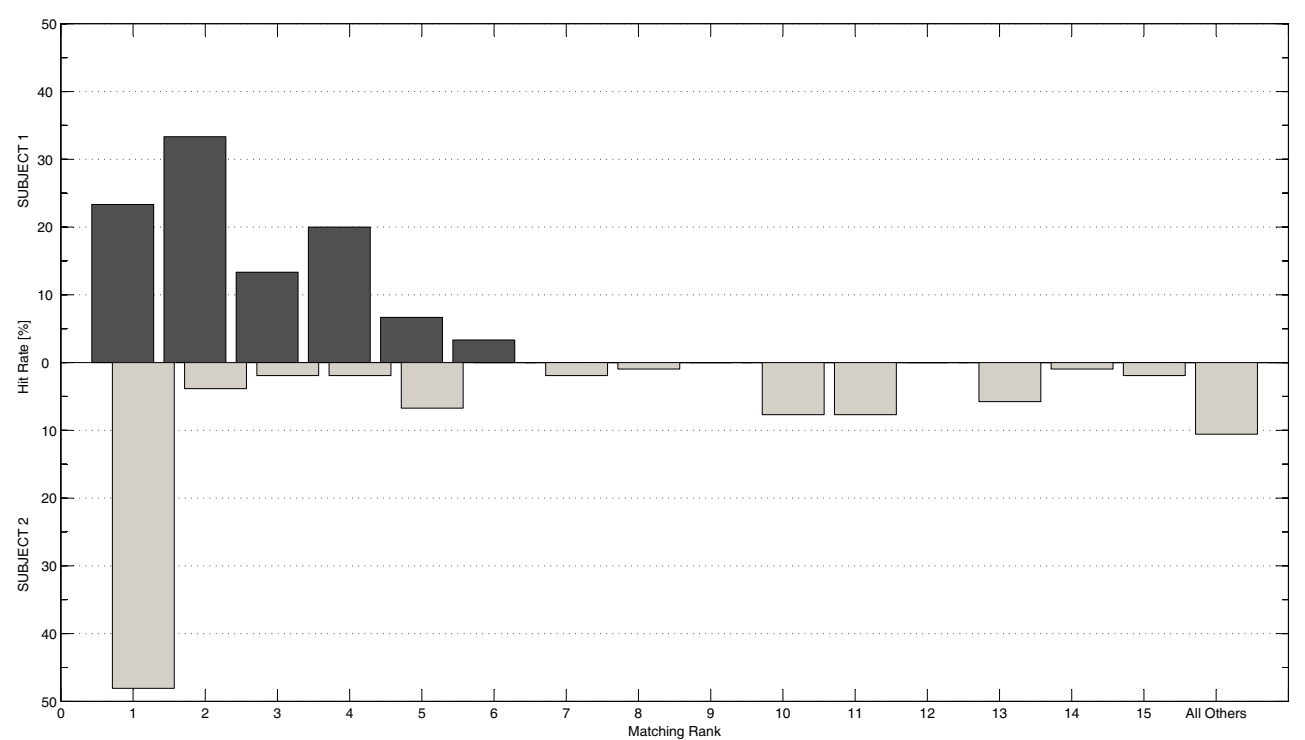

Figure 5. "Uniqueness-Test": Matching ranks for two test participants with 31 (dark grey) and 105 (light grey) samples. Results above rank 15 are merged into one class.

The primary conclusions are:

(i) Universality and (ii) Collectability: All utilized features are based on pressure patterns, acquired from a seat- and a backrest-mat (unobtrusivly integrated in a car seat). A driver has to sit on the seat, therefore both of the features universality and collectability can be guaranteed.

(iii) Permanency: This feature gives evidence about system stability regarding "noise" caused by the driver (e.g. with different clothes, artefacts in the pockets, age or weight). Experiments confirmed our assumptions that the system is vulnerable to (large) objects in the back-pocket and to different clothes (ski overall vs. swimming trunks).

(iv) Uniqueness: Results from the test on uniqueness are rather poor. In the smaller experiment, exact matching between 31 test data sets and the database is given in $23.33 \%$, while deviation is very low (in any case, the comparison leads to classes 1 to 6 , with highest values on rank 2 and 1). A small value for the variance stands for high systems stability and "low noise". The second experiment on 105 data sets directs to a divergent result: Exact match is given in $48.08 \%$, the remaining $51.92 \%$ are dispersed from classes 2 to 30 , with $86.54 \%$ inside classes 1 to 10 .

Nevertheless, rework on algorithms on the basis of changed features should lead to major improvements, especially for the parameter uniqueness:

(i) Feature Vector Calculation: Execute comparison of parameters first, after that apply weight factors and finally calculate the feature vector (the other way around as now). (ii) High-Pressure Areas: Because of excessive loads on the mat for special regions (e.g. on pelvic bones), low- and mid-pressure areas should be used more extensivly.

(iii) Filters: Low-pass filters should be applied to acquired data before performing feature-evaluations.

\section{References}

[1] Driver distraction trends and issues. Computing 86 Control Engineering Journal, 16(1):28-30, 2005. ISSN: 0956-3385.

[2] G. Andreoni, G. C. Santambrogio, M. Rabuffetti, and A. Pedotti. Method for the analysis of posture and interface pressure of car drivers. volume 33, pages 511-522. Department of Bioengineering, Polytechnic of Milan, Plazza Leodnardo da Vinci and Centro di Bioingegnerio, Via Capecelatro, Milan, Italy, Elsievier Science Ltd., Applied Ergonomics, 2002.

[3] V. Coroama. The smart tachograph - individual accounting of traffic costs and its implications. In Proceedings of Pervasive 2006, pages 135-152, Dublin, Ireland, May 2006.

[4] V. Coroama and M. Langheinrich. Personalized vehicle insurance rates - a case for client-side personalization in ubiquitous computing. In Workshop on Privacy-Enhanced Personalization at CHI 2006, Montréal, Canada, Apr. 2006.

[5] European Commission, Directorate-General Information Society and Media, ICT for Transport. Intelligent car brochure. http://ec.europa.eu/information_society/ activities/intelligentcar/docs/right_column/ intelligent_car_brochure.pdf, 092007. 
[6] M. Ferguson-Pell. Pressure mapping systems. TeamRehab Report, pages 28 - 32, October 1992.

[7] E. Giles. Discriminant function sexing of the human skeleton. Personal Identification in Mass Disasters, pages 99-109, 1970. Smithsonian Institution. Washington, D.C.

[8] J. Healey and R. Picard. SmartCar: Detecting Driver Stress. Proceedings of 15th International Conference on Pattern Recognition (ICPR'00), 4:218-221 vol.4, 2000.

[9] A. Heide and K. Henning. The "cognitive car": A roadmap for research issues in the automotive sector. In Annual Reviews in Control, volume 30, pages 197 203. Department of Computer Science in Mechanical Engineering, RWTH Aachen University, Germany, Elsevier, September 2006.

[10] R. Ishiyama, H. Ikeda, and S. Sakamoto. A compact model of human postures extracting common motion from individual samples. In Proceedings of the 18th International Conference on Pattern Recognition (ICPR'06), page 4. Media and Information Research Laboratories, NEC Corporation, IEEE Computer Society Press, 2006.

[11] A. Jain, L. Hong, and S. Pankanti. Biometric identification. In Communications of the ACM, volume 43, pages 90-98, New York, NY, USA, 2000.

[12] H. Miller. Body support in the office: Sitting, seating, and low back pain. Technical report, Hermann Miller Inc., 2002.

[13] K. J. Overbeeke, P. Vink, and F. K. Cheung. The emotion-aware office chair. In Proceedings of Int. Conference on Affective Human Factors Design. Asian Academic Press, 2001.

[14] W. T. Phenice. A newly developed visual method of sexing the os pubis. American Journal of Physical Anthropology, 30:297-302, 1969.

[15] P. J. Philips, A. Martin, C. L. Wilson, and M. Przybocki. An introduction to evaluating biometric systems. IEEE Computer, pages 56-63, February 2000. National Institute of Technology.

[16] R. W. Picard, E. Vyzas, and J. Healey. Toward machine emotional intelligence: Analysis of affective physiological state. Technical report no. 536, MIT Media Laboratory, Perceptual Computing, 20 Ames Street, Cambridge, MA 02139, USA, 2001.

[17] T. D. Stewart. Sex determination of the skeleton by guess and by measurement. American Journal of Physical Anthropology, 12:385-392, 1954.

[18] L. Stibor and Y. Zang. Schutzengel 2010: Fahrzeugkommunikation schafft Sicherheitsreserven. c't magazin für Computertechnik, 19:170-173, 2007.

[19] M. Zhu, A. M. Martinez, and H. Z. Tan.

Template-based recognition of static sitting postures. In Proceedings of The Workshop on Computer Vision and Pattern Recognition for Human Computer Interaction, held at the IEEE Conference on Computer Vision and Pattern Recognition (CVPR'03), page 6 pp. Dept. of Electrical Engineering, The Ohio State University and Purdue
University, IEEE Computer Society, Madison, Wisconsin, June 172003. 